\title{
Polygraph testing of sex offenders in a high secure hospital
}

\author{
David K. Ho, ${ }^{1}$ Nikki Collins, ${ }^{1}$ Morris Vinestock, ${ }^{1}$ Mrigendra Das ${ }^{1}$
}

The Psychiatrist (2013), 37, 141-143, doi: 10.1192/pb.bp.112.038463

${ }^{1}$ Broadmoor Hospital, Crowthorne, UK

Correspondence to David K. Ho (email: david.ho@nhs.net)

First received 13 Jan 2012, final revision

7 Nov 2012, accepted 9 Nov 2012
Aims and method To illustrate the clinical benefit of polygraph testing for mentally disordered sex offenders at a high secure psychiatric hospital. It is a retrospective review of two patients' case notes and of interviews with clinicians. Post Conviction Sex Offender Testing (PSCOT) was used to assist these patients in making disclosures regarding their sexual history and to aid their treatment.

Results Post Conviction Sex Offender Testing was responsible for bringing about new disclosures relating to the patients' sexual histories and aiding their treatment progression to lower secure settings. New information was incorporated into the patients' treatment programmes and risk management plans. Post Conviction Sex Offender Testing also encouraged a more honest and effective participation in sex offender treatment programmes and allowed the evaluation of antilibidinal medication.

Clinical implications Post Conviction Sex Offender Testing should be more widely considered among mentally disordered sex offenders who have been challenging to treat, as it has the potential to aid their management and progression to lower-security settings.

Declaration of interest None.
The modern polygraph has primarily been used to detect deception by identifying changes in physiological parameters (e.g. respiration, pulse rate) which are triggered by a 'stress response' to specific questions. This association has been known for hundreds of years and is embedded in both historical and fictional literature. ${ }^{1-3}$

Increasingly, polygraph testing is also being considered as a useful tool to aid disclosures among sex offenders. ${ }^{4-7}$ This technique is known as post-conviction sex offender testing (PCSOT). A recent large-scale review by the UK Ministry of Justice ${ }^{4}$ found that polygraph testing increased the chances of sexual offenders revealing information - or making clinically significant disclosures - which in turn aided their management, supervision, treatment and risk assessment.

This report aims to illustrate the clinical benefit of polygraph testing for mentally disordered sex offenders. It tells the story of two patients at a high secure psychiatric hospital who have, in the past, been difficult to treat. They also posed a significant risk to the public of serious sexual reoffending, which hindered their progression to lower levels of security. They were tested using the PCSOT, to assist them in making disclosures regarding their sexual history and to aid their treatment and management in less secure psychiatric settings.

\section{Method}

We report on two cases where polygraph testing was used. In both cases the patients had extensive histories of sex offending and were being treated in a high secure psychiatric hospital. They were assessed by their consultant psychiatrist and were found to have the capacity to make an informed decision, and they consented to the procedure. Before the testing, the patients received clear and detailed information about PCSOT.

For disclosures that were potentially incriminating, the standard disclosure procedure for the hospital was followed whereby any relevant information was brought to the clinical team and the relevant authorities were notified if deemed appropriate. This formed part of the consent form.

The case notes and polygraph results were reviewed retrospectively. The patients' progress was also discussed with the relevant clinical teams. The data collection and reporting was anonymised to preserve patient confidentiality.

\section{Polygraph procedure}

The comparison question technique was used for the polygraph testing. This is the most researched technique in post-conviction settings with sex offenders. It includes three types of questions: relevant questions about issues of 
clinical importance; irrelevant questions about other issues; and comparison questions, designed to encourage the interviewee to lie and thus to elicit autonomic arousal responses, such as increased respiration and pulse rate that are indicative of deception.

The specific type of PCSOT used was the sexual history disclosure examination, which is designed to gather and verify information about an individual's complete sexual history. The examination comprises four key elements. ${ }^{8}$ The first is a pre-test interview that focuses on reviewing in detail the interviewee's sexual history and formulating and reviewing test questions. During this phase the interviewee is instructed to tell a deliberate lie for him to be acquainted with the instrument and to establish a baseline of their physiological responses.

The second element is the actual polygraph test, which has approximately 10-12 questions (4 are relevant to the test issue) that are each asked 3-5 times. The third element is a detailed scoring of the charts, followed finally by the post-test interview to feed back results and clarify any outstanding issues.

The equipment used to measure the patients' physiological responses was a Lafayette LX4000 device, which recorded cardiac activity, galvanic skin response and breathing. In addition, a motion sensor pad on the interviewee's chair recorded any movement (to detect possible employment of counter-measures).

\section{Results}

\section{Case 1: patient $\mathbf{A}$}

Patient A has a 20-year history of care in secure psychiatric hospitals, including 10 years in a high secure setting. He has a diagnosis of dissocial personality disorder and has been convicted for repetitive sexual assaults on children. His index offence was the indecent assault of a child, which was committed while he was on a trial period of leave, on his way to a community sex offender treatment group.

During his time in hospital patient A has completed various individual and group psychological therapies and an 18-month sex offender treatment programme. A polygraph examination was conducted when the patient was being considered for transfer to a medium secure hospital. The purpose was to establish his sexual history and current baseline sexual behaviours to aid his rehabilitation in this new setting. Specifically, the test aimed to determine whether he had any inappropriate sexual fantasies of children or had masturbated to images of children.

The test results indicated deception in the areas of continued fantasy and masturbation to images of children. Further significant disclosures pertaining to his risk profile were also obtained. These include past practices of 'cruising' - looking for children in vulnerable areas such as schools, masturbation in children's play parks, and voyeurism in the community. He also revealed other beliefs indicative of his sexual deviancy, for instance, the patient stated that 'anything less than penetrative sex was OK and you did not need permission for it'.

The information disclosed during the polygraph test allowed for a more focused individual psychological therapy and overall clinical assessment of change in his negative attitudes. It was also incorporated into his HCR-20 risk assessment, which enabled the receiving medium secure unit to appropriately manage his risk. Following this initial test, patient A was treated with triptorelin, an antilibidinal medication. Subsequent monitoring of his baseline sexual behaviours using PCSOT 6 months later revealed significantly less deception. Patient A has now been granted trial leave to a medium secure psychiatric unit.

\section{Case 2: patient B}

Patient B has a history of dissocial and borderline personality disorder with features of psychopathy. He has been convicted of repeated counts of arson and his index offence involved arson to property with accompanying sexual self-gratification. He claimed that as a teenager the police cautioned him for perpetrating sexual assaults on children, yet he has not been convicted of any sexual offence and therefore has not qualified for formal sex offender therapy. His management is complicated further by his frequently inconsistent reporting of his sexual history, fantasies and beliefs. For instance, patient B reported that he had been a victim of child sexual abuse, however, he retracted that statement some time afterwards. A polygraph test was requested to gain clarification regarding his sexual history, explore disclosures regarding his reported sexual assaults, and determine future risk issues.

The test revealed deception in the area of sexual contact with children. He disclosed that he had had sexual contact with his sibling prior to the index offence. This was relevant, as he had previously denied any sexual contact with his sibling in this period. Patient $\mathrm{B}$ also disclosed that he had inappropriate contact with a female baby when he was a teenager.

In this case, the process of polygraph testing was instrumental in bringing about disclosures of information concerning important issues that the patient had previously denied. The additional disclosure of potential risk to children and family members are crucial factors to be taken into account when planning for his supervision in the community (e.g. by Multi-Agency Public Protection Arrangements (MAPPA) and probation services). Following the test, the receiving medium secure unit felt more confident managing these known risks and the patient has since been referred to them.

\section{Discussion}

To our knowledge, this is the first report on the use of PCSOT within high secure psychiatric services. We find that for both case studies the tests were responsible for bringing about new disclosures relating to the patients' sexual histories, which in turn helped strengthen their rehabilitation (through targeted psychological therapies or medication) and management in medium secure settings.

The PCSOT polygraph test has been shown to provide particular benefit in identifying previously unknown risk patterns. This is also found in the literature. For instance, English et $a l^{9}$ reported a study of 232 adult sex offenders in 
the community where PCSOT was used as an adjunct to supervision. They found that polygraph testing provided information about additional types of victims who were at risk, identified new crimes and detected high-risk deviant behaviours. It is of significance that PCSOT has the potential to identify these 'crossover' patterns of sexual offending, for example across victim gender or age, which is known to be prevalent among convicted adult sex offenders. ${ }^{1}$

Any negative attitudes or deviant behaviours detected through PCSOT could also be used as markers of change within psychological therapy, and in evaluating the impact of pharmacological treatment. These same markers can be monitored and supervised as the patient progresses to a lower-security environment and eventually the community, as illustrated in the case of patient A.

A further benefit of PCSOT is that it encourages more honest participation in psychologically based therapies. Currently, the majority of treatment regimes for sex offenders are psychosocial, including the sex offender treatment programme which is the standard treatment in England and Wales. ${ }^{10,11}$ However, current evidence for its efficacy in reducing sexual recidivism is lacking, and one possible reason for this may be a reluctance of participants to reveal clandestine thinking patterns and to change deviant behaviour. ${ }^{11}$ Offenders may complete this therapy without meaningful participation, especially if treatment has been imposed on them through criminal justice or healthcare provisions. If, however, patients are encouraged to reveal previously hidden information through PCSOT, as with patient B, psychological therapies can be better targeted to address specific behaviours.

Further, once revealed, the offender may feel that he has 'nothing more to lose', providing a mental impetus away from concealing these desires and encouraging him to work through the treatment programme in a more honest manner.

Polygraph testing has been controversial since its conception and its use for PCSOT has also received some scepticism. Key criticisms of polygraph testing relate to the instrument's accuracy and validity, and to non-standardised testing procedures or questioning methods used during testing. ${ }^{12,13}$ However, the US National Academy of Sciences place the test's accuracy at between 80 and $90 \%$ and conclude that "polygraph tests can discriminate lying from truth telling at rates well above chance, though well below perfection'. ${ }^{14}$

There is also a growing evidence base proving the value of the test in relation to assisting disclosures. ${ }^{4-6,15}$ Although past studies have mostly focused on voluntary participants, representing a small sample of particularly motivated people, more recent publications have demonstrated the positive effects of compulsory testing for sex offenders. ${ }^{4,16}$ Further research is required to ascertain the impact of the polygraph test among mentally disordered populations. This needs to take into account complex ethical issues regarding mandatory testing of patients, their ability to consent, and what is done with potentially incriminating information.

\section{The usefulness of polygraph testing}

The case studies presented in this paper contribute to a growing body of evidence that PCSOT aids clinically significant disclosures. In these examples, the tests helped to identify previously unknown disclosures regarding deviant thought patterns and past sexual offences, and this new information was incorporated into the patients' treatment programmes and risk management plans. Its use encouraged a more honest and effective participation, which is essential in sex offender treatment programmes. For patient $\mathrm{A}$, the information gathered through PCSOT also helped to develop a baseline understanding of his behaviour to monitor the effectiveness of antilibidinal medication.

We recommend that PCSOT should be considered more widely among patients who have been challenging to treat, as it has shown to aid their management and progression to lower-security settings.

\section{About the authors}

Dr David K. Ho, specialist registrar in forensic psychiatry, Ms Nikki Collins psychologist, Dr Morris Vinestock, consultant, Dr Mrigendra Das, consultant, all at Broadmoor Hospital, West London Mental Health NHS Trust, Crowthorne, Berkshire, UK.

\section{References}

1 Grubin D, Madsen L. Lie detection and the polygraph: a historical review. Br J Forensic Psychiatry Psychol 2005; 16: 357-69.

2 Segrave K. Lie Detectors: A Social History. McFarland and Company, 2004

3 Sullivan E. The Concise Book of Lying. Picador, 2001.

4 Gannon TA, Wood J, Pina A, Vasquez E, Fraser I. The Evaluation of the Mandatory Polygraph Pilot (Ministry of Justice Research Series 14/12). Ministry of Justice, 2012.

5 English K, Pullen S, Jones L. Managing Adult Sex Offenders on Probation and Parole: A Containment Approach. American Probation and Parole Association, 1996

6 Grubin D. The case for polygraph testing of sex offenders. Legal Criminol Psychol 2008; 13: 177-89.

7 Wilcox DT. Application of the clinical polygraph examination to the assessment, treatment and monitoring of sex offenders. J Sex Aggression 2000; 5: 134-52.

8 Kleiner M. Handbook of Polygraph Testing. Academic Press, 2002.

9 English K, Jones L, Pasini-Hill D, Patrick D, Cooley-Towell S. The Value of Polygraph Testing in Sex Offender Management (Research Report Submitted to the National Institute of Justice). Colorado Department of Public Safety, Division of Criminal Justice, Office of Research and Statistics, 2000.

10 Hanson RK, Gordon A, Harris AJR, Marques JK, Murphy W, Quinsey VL, et al. First report of the collaborative outcome data project on the effectiveness of treatment for sex offenders. Sex Abuse 2002; 14: 169-94.

11 Ho DK, Ross CC. Cognitive behavioural therapy for sex offenders. Too good to be true? Crim Behav Ment Health 2012; 22: 1-6.

12 Ben-Shakhar $\mathrm{G}$. The case against the use of polygraph examinations to monitor postconviction sex offenders. Legal Criminol Psychol 2008; 13: 191-207.

13 Meijer EH, Verschuere B, Merckelbach HLGJ, Crombez G. Sex offender management using the polygraph: a critical review. Int J Law Psychiatry 2008; 31: 423-9.

14 National Research Council. The Polygraph and Lie Detection. National Academic Press, 2003.

15 Grubin D. The potential use of polygraphy in forensic psychiatry. Crim Behav Ment Health 2002; 12: S45-53.

16 Grubin D, Madsen L, Parsons S, Sosnowski D, Warberg B. A prospective study of the impact of polygraphy on high-risk behaviors in adult sex offenders. Sex Abuse 2004; 16: 209-22. 